\section{Aktivierende Therapien bei Parkinson-Syndromen}

\author{
A. O. Ceballos-Baumann; F. E. Schroeteler; U. M. Fietzek; K. Ziegler
}

Schön Klinik München-Schwabing, Abt. für Neurologie und klinische Neurophysiologie mit Parkinson-Fachklinik

\section{Schlüsselwörter}

Parkinson-Syndrome, Physiotherapie, Ergotherapie, Schluck-, Stimm- und Sprechtherapie, Cues, Freezing

\section{Zusammenfassung}

Trotz wachsender Möglichkeiten in der Pharmakotherapie und der tiefen Hirnstimulation können bei der Mehrzahl der Parkinson-Patienten im Verlauf wesentliche Funktionen wie Gleichgewicht, Gehen, Sprechen, Schlucken und Kognition langfristig nur unzureichend gebessert werden. Den aktivierenden Therapien wie Physio-, Sprech-, Schluck- und Ergotherapie kommt hierbei eine zunehmende Rolle zu. Der Empfehlungsgrad in den S3-Leitlinien erreicht für die Haupttherapieformen Level A (Physiotherapie) und B (Logopädie, Schlucktherapie, Ergotherapie). Für die Physiotherapie finden sich die meisten Studien. Neue Ansätze mit Krafttraining, Tanzen, Tai Chi sowie gerätegestützte Ansätze wie Laufbandtraining kombiniert mit neuen Entwicklungen wie virtueller Realität und Spielkonsolen („Exergaming") weisen eine gute Studienevidenz auf. Die S3-Leitlinie der DGN fordert für alle Patienten in allen Stadien Zugang zu einer zeitlich ausreichenden Physiotherapie. Dieser CME Artikel fasst den Stand der aktivierenden Therapien in der Langzeitversorgung von Parkinson-Patienten zusammen.

\section{Keywords}

Parkinson's disease, physiotherapy, occupational, swallowing, voice and speech therapy, cues, freezing

\section{Summary}

Despite advances in pharmacotherapy and deep brain stimulation most patients with Parkinson's disease (PD) experience impairments in balance, gait, speech, voice, swallowing and cognition. Non-pharmacological ("Activating") therapies such as physiotherapy, occupational, swallowing, voice and speech therapy play an important role in alleviating these symptoms. The recommendation level in the German guidelines is the highest for physiotherapy, for occupational and speech therapy it is second highest. Most trials have been performed in physiotherapy. New approaches with progressive resistance training, weight lifting, dance, Tai chi, thread mills combined with virtual reality and exergaming show good evidence in randomised trials. The German S3 guidelines demand that all PD patients in all stages have access to sufficient time resources of physiotherapy. This CME article summarises the role of activating interventions in the long term care of PD patients.

Non-pharmacological therapies in Parkinsonian syndromes

Nervenheilkunde 2018; 37: 264-271

eingegangen am: 20. Februar 2018

angenommen am: 5. März 2018
Mit dem Begriff „aktivierende Therapien“ (1) wird ein heterogenes Spektrum von Interventionen bezeichnet, die klassischerweise im Rahmen der Physiotherapie, Ergotherapie und Logopädie (Stimm-, Sprech- und Schlucktherapie) zur Anwendung kommen. Dazu gehören unter anderem sportliches Training, Krafttraining, Nordic Walking, Tanzen, Musik-und Theatertherapie, Tai Chi, Qi Gong, gerätegestützte Ansätze wie Laufbandtraining, Programme, die Spielkonsolen wie z. B. Wii („Exergaming“) und virtuelle Realität einsetzen. Psychotherapie nicht, aber psychologische Interventionen wie kognitives Training können auch dazu gezählt werden (2). Kognitives Training speziell bei Parkinson entwickelt sich gerade und wird in dieser Übersicht nicht behandelt.

Unter den aktivierenden Therapien hat die Physiotherapie bei den Behandlungspräferenzen von Medizinern und Patienten schon vor Einführung der Dopaminersatztherapie einen hohen Stellenwert, wenngleich anstatt eines problemzentrierten Trainings häufig ungezielt Therapien verordnet werden, die nicht aktivierend sind, sondern sogar passiv. Krankengymnastik nach Bobath kommt beispielsweise viel zur Anwendung, obwohl diese nicht für Parkinson-spezifische motorische Probleme, sondern für die Spastik konzipiert wurde. Außerdem wurde gezeigt, dass das in vielen Gesundheitssystemen übliche niederschwellige Training bei Parkinson wenig wirksam ist (3).

Mittlerweile gibt es auch für andersartig schwer zu behandelnde Parkinson-Symptome (z. B. Gleichgewichtsstörungen, motorische Blockaden wie Freezing, Dysarthrophonie) spezifische neurophysiologisch begründete und wissenschaftlich gut evaluierte aktivierende Therapien. Patienten mit Parkinson profitieren von physiotherapeutischem Training insbesondere in 
Mobilität und Alltagskompetenz (4). Körperliches Training hat außerdem positive Effekte auf motorische und nicht motorische Symptome wie Kognition (5), Depression (6) und Schlaf (7).

Aus grundlagenwissenschaftlicher Sicht ergibt sich eine klare Rationale für aktivierende Therapien. Tierexperimentelle Studien haben gezeigt, dass körperliches Training die Produktion von Wachstumsfaktoren fördert und synaptische Übertragung sowie Neuroplastizität einschließlich der Expression von Dopaminrezeptoren im präfrontalen Kortex verbessern kann $(8,9)$. Darüber hinaus weist körperliche Aktivität einen protektiven Effekt nicht nur auf die kardiovaskuläre Morbidität und Mortalität, sondern auch auf die spätere Entwicklung eines Morbus Parkinson. Seit den 1990erJahren gibt es Daten, die eine ParkinsonRisikoreduktion in Abhängigkeit von dem Ausmaß körperlicher/sportlicher Aktivität im Erwachsenenalter nahelegen, z. B. nach einer Beobachtung von über 50000 Akademikern, die während des Studiums und danach sich sportlich betätigten (10). In einer Krebsvorbeugungsstudie mit 43000 Schweden, die von 1997 bis 2010 beobachtet wurden, zeigte sich, dass 6 Stunden Bewegung pro Woche das Parkinson-Risiko um bis zu 45\% reduziert (11). Zusammen mit weiteren prospektiven Kohortenstuchen Daten ist die Konsequenz, dass schon in frühen Phasen der Krankheit mit Training beginnen werden sollte. Es gibt noch einen weiteren entscheidenden Grund, frühzeitig mit aktivierenden Therapien zu beginnen: Die Vorbeugung des „Teufelskreis“ der Inaktivität, die nicht nur die Folgen der Krankheit verstärkt, sondern auch mit der Entwicklung von Diabetes Typ 2 und Adipositas vergesellschaftet ist (12).

Trotz laufender Neuentwicklungen im medikamentösen und neurochirurgischen Bereich, ist es nicht nur die begrenzte Wirksamkeit, sondern bei späten Stadien der Parkinson-Krankheit und atypischen Parkinson-Syndromen auch die unzureichende Verträglichkeit der pharmakologischen und operativen Therapie, die ein integriertes Behandlungskonzept unter Einbeziehung der aktivierenden Therapien erforderlich machen. Nach ca. 15 Jahren Verlauf eines typischen idiopathischen Parkindien und den grundlagenwissenschaftli-

son-Syndroms (IPS) unter optimaler kontrollierter Therapie dominieren die auf Dopaminergika nicht ansprechende Symptome: Es stürzen $81 \%$ der Patienten, $23 \%$ ziehen sich dabei Frakturen zu, 80\% haben eine beeinträchtigende Dysarthrophonie und $50 \%$ verschlucken sich regelmäßig (13) ( $>$ Tab. 1).

Die S3-Leitlinie der DGN fordert für alle Patienten in allen Stadien Zugang zu einer zeitlich ausreichenden Physiotherapie (14). Aktuelle Auswertungen epidemiologischer Daten zum Morbus Parkinson in Deutschland (MoPED) zeigen jedoch, dass in einer großen Gruppe von knapp 22000 Patienten im Jahre 2015 nur 36\% überhaupt physiotherapeutisch betreut waren. Der Empfehlungsgrad in den S3-Leitlinien für Physiotherapie entspricht dem höchsten Empfehlungs- und Evidenzgrad wie bei Dopaminergika. Für Logopädie und Ergotherapie fällt bisher der Empfehlungsgrad niedriger als bei der Physiotherapie aus (Patienten „sollten“ anstatt „sollen“ Zugang zu ergotherapeutischer, bzw. logopädischer Behandlung haben).

\section{Spektrum aktivierender Therapien bei Parkinson-Syndromen}

Eine zunehmende Auswahl an aktivierenden Therapien steht zur Verfügung ( $>$ Tab. 1). Die wissenschaftliche Evidenz für diese Methoden wächst. Am besten erwiesen sind die Interventionen, die Physiotherapie und körperliches Training beinhalten. Vielleicht liegt das daran, weil physiotherapeutische im Vergleich zu logopädischen und ergotherapeutischen Interventionen am meisten untersucht wurden und methodisch leichter erfasst werden können.

\section{Tab. 1}

Levodoparesistente

Symptome in späteren Stadien der ParkinsonKrankheit
Ansätze wie Tai Chi, die früher als „alternativ“ galten, werden in den „Werkzeugkasten" der aktivierenden Therapien mit aufgenommen und können bei vorhandenen Ressourcen (d. h. erfahrene Therapeuten) und Akzeptanz/Motivation der Patienten angeboten werden. Tai Chi wurde z. B. bei 195 Patienten mit Kontrollinterventionen (Kraft- bzw. Dehnungstraining) untersucht und höchstranging veröffentlicht (15). Bei einer „Dosis“ von 2-mal pro Woche 60 Minuten über einen Zeitraum von 6 Monaten zeigten sich signifikante Verbesserungen korrektiver und proaktiver Gleichgewichtsleistungen sowie eine Reduzierung der Sturzrate neben anderen Parametern. Die Kontrollintervention der Dehnungsübungen verzeichnete durchgehend keine messbaren Verbesserungen (15).

Künstlerische Therapien wie Musiktherapie und Tanzen haben schon lange eine große Akzeptanz. Die rhythmisch-akustischen Stimulation (RAS) zur Verbesserung von Gehgeschwindigkeit, Schrittlänge und Gangblockaden mit Musik im Vergleich zu ohne Musik könnte eine höhere Patientenzufriedenheit mit sich bringen. Das therapeutische Singen wurde in Pilotstudien als Gruppenbehandlung evaluiert (16-18). Für die Tanztherapie gibt es die besten Daten für den argentinischen Tango (19). Zunehmend werden abgewandelte Computerspiele und virtuelle Realität bei den aktivierenden Therapien eingesetzt. Virtuelle Realität und Exergaming bieten ein Training in einem stimulierenderem und abwechslungsreicherem Umfeld an, als es herkömmliche Verfahren im klinischen Alltag können ( $>$ Abb. 1). Unterstützt wird der Lerneffekt durch den Spaß am Spiel (20).

Praktische Übersichten sind die „Europäischen Physiotherapie-Leitlinie zum idiopathischen Parkinson-Syndrom" (21) in deutscher Sprache und die auf Englisch 


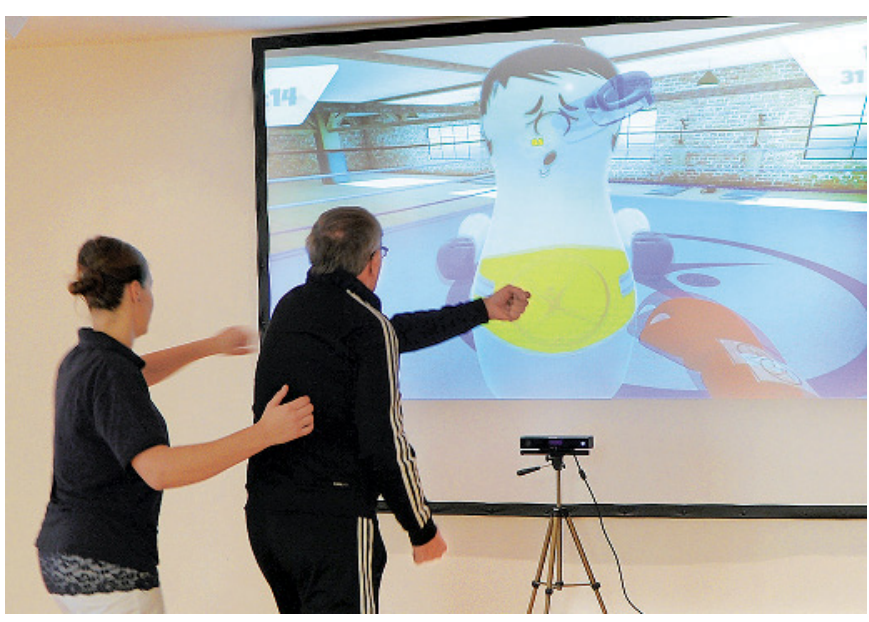

Abb. 1

Die Abbildung zeigt einen Patienten beim virtuellen Boxen. Mit diesem "Spiel" werden großamplitudige Bewegungen, Gleichgewicht und Kraft mit hoher Motivation bei beiden Geschlechtern trainiert. verfügbaren niederländischen Leitlinien für Ergotherapie (22) und Logopädie (23) (www.ParkinsonNet.info). Darin werden Behandlungsansätze für den gesamten Krankheitsverlauf, spezifische Assessments und effektive Therapiemethoden für viele auf Medikamente unzureichend ansprechende Probleme bei Parkinson-Syndrome vorgestellt.

\section{Indikationsstellung für aktivierende Therapien}

Gemäß den deutschen S3-Leitlinien (14) und den europäischen Leitlinien für Physiotherapie bei Parkinson (21), aber auch den britischen NICE (24) lassen sich Stadien abhängig folgende Indikationen für den Einsatz von aktivierenden Therapien herleiten:

\section{Diagnosestellung}

- Inaktivität vorbeugen,

- körperliche Leistungsfähigkeit steigern,

- Sturz- und Bewegungsangst vermeiden,

- Unterstützung im Selbstmanagement.

\section{Weiterer Verlauf}

- Verbesserung/Erhalt von aerober Kapazität, Bewegungsamplituden, Gleichgewicht, Bewegungsinitiierung (Freezing), Transfers,

- Sturzprävention,
- Erhalt der beruflichen und familiären Rollen, des Arbeitsplatzes häuslicher Versorgung und Freizeitaktivitäten,

- Verbesserung/Erhalt von Autonomie bei Basis-ADL (wie Essen, Trinken, Waschen und Ankleiden) und Aktivitäten (wie Küchen-, Haushalts- und खEinkaufsaktivitäten),

- Verbesserung von Sicherheit für motorische Aktivität. Bei sturzgefährdeten Patienten sollte idealerweise mit einem Hausbesuch (Ergo-/ Physiotherapie), die Verletzungsmöglichkeiten in der häuslichen Umgebung reduziert werden, Umbaumaßnahmen und Hilfsmittelversorgung, z. B. mit einem Rollator eingeleitet werden.

- Verbesserung/Erhalt von Stimmlautstärke und Tonumfang,

- Erhalt von Kommunikationsfähigkeit, evtl. bis hin $\mathrm{zu}$ computergestützten Kommunikatoren,

- Verbesserung/Erhalt von Schlucken, insbesondere bei erhöhtem Aspirationsrisiko. Die Indikation und Effektivität der Therapie kann mittels flexible endoskopischer Evaluation des Schluckaktes (FEES) am besten beurteilt werden.

Bei der Befunderhebung sollte wegen der Dringlichkeit der Indikationsstellung besonders auf Symptome geachtet werden, die mit einem erhöhten Risiko für Sekundärkomplikationen wie Stürze und Dysphagie behaftet sind und entsprechende Intervention bereits zu Therapiebeginn erforderlich machen. Gerade bei atypischen Parkinson-Syndromen sollte die Ergothe- rapie möglichst früh im Krankheitsverlauf eingesetzt werden, um Selbstständigkeit zu erhalten (25). Mit Fortschreiten der Krankheit sind symptomspezifische Therapieansätze indiziert. Erreichte Besserungen bleiben nur bei kontinuierlichem Training länger bestehen, so wie bei Medikamenten die auch kontinuierlich eingenommen werden müssen.

Prinzipiell gilt für eine effektive Indikationsstellung bei fluktuierenden Patienten, dass die Therapiezeiten in die Phasen des medikamentösen On gelegt werden, da die Übungsfähigkeit und die Frustrationstoleranz in den Off-Phasen stark reduziert sein können (26). Gegebenenfalls sollte von schnell wirksamer dopaminerger Bedarfsmedikation (Levodopa, Apomorphin subkutan) Gebrauch gemacht werden, um einen On-Zustand vor/während der Therapie zu erreichen.

Die Therapieoptionen sind mannigfaltig. Das beinhaltet auf der wissenschaftlichen Ebene das Problem, dass es schwer ist, viele der einzelnen Behandlungsansätze bei großen Kollektiven hinsichtlich ihres Nutzens miteinander zu vergleichen. Auf der Versorgungsebene ist es schwer, genügende, mit den verschiedenen Ansätzen kundige Therapeuten $\mathrm{zu}$ finden und eine entsprechende Therapiequalität über viele Verfahren hinweg zu gewährleiten. Andererseits kann der Therapeut aus einem „Werkzeugkasten“ auf viele evaluierte Therapieverfahren zurückgreifen und eine seinen Möglichkeiten und den Bedürfnissen des Patienten personalisierte Therapie gestalten.

\section{Physiotherapie}

Schon lange ist klinisch bekannt, dass sich Parkinson-Patienten mit externen sensorische Hinweisreizen aus ihren motorischen Blockade beim Gehen („Freezing of gait“) lösen können ( Tab. 3). Cueing (engl. cueing, dt. Hinweisreize geben) ist definiert als Angebot räumlicher oder zeitlicher, externer Reize, die helfen Gehen zu initiieren und zu fazilitieren. In einer multinationalen konnte gezeigt werden, dass Cueing auch in der häuslichen Umgebung effektiv nutzbar ist (27). Wichtig ist für den jeweiligen Patienten geeignete Cues zu identifi- 
zieren und deren Anwendung - auch unter Einbindung der Angehörigen - intensiv in den entsprechenden Alltagssituationen zu trainieren. Evaluierte, strukturierte Programme wie das Münchner Antifreezing Training (MAFT) wurden entwickelt (28-31).

\section{Virtuelle Realität (VR) in der Therapie der Gangstörung}

Laufbandtraining wird mit VR kombiniert ( Abb. 3). Simples Laufbandtraining alleine ist effektiv, bei Parkinson-Patienten Schrittlänge, Gehtempo und -strecke zu steigern. Mit VR werden zusätzlich z. B. Hindernisse wie Hürden oder Pfützen, Passanten und Autos über einen Bildschirm oder Projektion auf das Laufband projiziert. Durch Veränderung der „Umweltfaktoren “ kann die Komplexität individuell erhöht werden. So werden während des Gehens gleichzeitig motorische und kognitive Aspekte des Sturzrisikos trainiert (32). In einer großen Studie mit jeweils ca. 100 Parkinson-Patienten, Patienten mit „mild cognitive impairment" und altersentsprechenden Kontrollen konnte damit die Sturzfrequenz auch über die 6 Monate des Trainings (2x30 min Woche) gesenkt werden (33).

\section{Gleichgewicht}

Repetitives, selektives Training von Schutzschritten, d. h. kompensatorischen Ausgleichsschritten durch destabilisierende Reize („Schubs-Training“) vergrößerte in einem Zeitraum von zwei Wochen mit einer Trainingsfrequenz von 2-mal zwanzig Minuten pro Tag die Länge des ersten Schutzschrittes und verminderte die Reaktionszeit bis zur Bewegungsinitiierung des ersten Schutzschrittes (34). Parallel dazu verbessern sich auch die Gangparameter wie Schrittlänge und Schrittkadenz (Schritte pro Minute).

\section{Exergaming zur Therapie des Gleichgewichts}

Exergames sind Computerspiele, die durch grobmotorische Bewegungen des Spielers gesteuert werden. Videospiel und Echtzeitbewegungserfassung werden kombiniert
Tab. 2 Auswahl an aktivierenden Therapien

\begin{tabular}{|c|c|}
\hline Interventionen & zur Besserung von \\
\hline $\begin{array}{l}\text { Physiotherapie } \\
\text { - aerobes Training } \\
\text { - Training von Kraft, großen Bewegungsampli- } \\
\text { tuden, Gleichgewicht, Anti-Freezing, ... } \\
\text { - Tai Chi, Exergaming, VR ... } \\
\text { Künstlerische Therapien: Tanz-, Musik-, Sing-, } \\
\text { Theater-, Kunsttherapie ... }\end{array}$ & $\begin{array}{l}\text { Bewegungsqualität- und Sicherheit, Selbststän- } \\
\text { digkeit, allgemeine Fitness, Minimierung sekun- } \\
\text { därer Komplikationen, positive Effekte auf nicht } \\
\text { motorische Funktionen wie Depression und Fati- } \\
\text { gue }\end{array}$ \\
\hline Sprech- und Schlucktherapie (Logopädie) & Kommunikation und Nahrungsaufnahme \\
\hline Ergotherapie & $\begin{array}{l}\text { Partizipation, Selbstständigkeit } \\
\text { (z. B. Anziehtraining) }\end{array}$ \\
\hline $\begin{array}{l}\text { psychologische Behandlung, kognitives Training, } \\
\text { Entspannungsverfahren, Computer-Training, VR, } \\
\text { Exergaming }\end{array}$ & $\begin{array}{l}\text { Kognition, Progredienzangst, Krankheitsbewälti- } \\
\text { gung, Affekt }\end{array}$ \\
\hline
\end{tabular}

und motivieren Patienten so zu messbarer, körperlicher Aktivität (20). Zum Einsatz kommen v. a. die Nintendo Wii Konsole mit den Balance-Spielen aus dem Wii Fit Paket sowie in einer Untersuchung die Kinect Xbox von Microsoft mit dem Spiel Kinect Adventures! $!^{\mathrm{TM}}$ ( $>$ Abb. 2).

In einer Metaanalyse wurde untersucht, ob Gleichgewichtsspiele (z. B. Kugelballett, Skislalom, Sportimitationen wie Tennis, Boxen, Bowling) ungefährlich, leicht durchführbar und effektiv waren. Die Balancefähigkeit und andere motorische Symptome verbesserten sich. In der einzigen randomisierten Kontrollstudie zur Effektivität von Nintendo Wii, waren Gleichgewichtsspiele und stationäres Gehen auf der Konsole einem herkömmlichen Balan-

Tab. 3 Training mit sensorischen Hinweisreizen, Triggern (engl. Cueing)
- Visuell
- Markierungen auf dem Boden
- Bewegung vor dem Spiegel
- Hindernisse
- Bodenunebenheiten
- Laser-Schuhe (>Abb. 2)
- Akustisch
- Metronom
- Klatschen
- Rhythmische Musik
- Kommandos
- Zählen
- Haptisch
- Geführte Bewegungen
- Taktile Stimulation

cetraining mit den gleichen Bewegungen aber ohne Feedback und externen Reizen, nicht überlegen. Unklar blieb, ob der Spaß der gleiche war (35).

\section{Kraft- und Ausdauertraining}

Bei einer 24-monatigen Studie (2 x 60 Minuten/Woche) eines Krafttrainings am Gerät von Knie-, Hüft-, Rückenextensoren, Ellenbogenflexoren- und -extensoren sowie der Schultergürtelmuskulatur mit steigenden Widerständen und zunehmendem Bewegungstempo wurde die ParkinsonSymptomatik im Vergleich zur Kontrollintervention im Off (ohne Medikamente) um 7 Punkte auf der Parkinson-Beurteilungsskala (UPDRS) gebessert (36). Das intensive Langzeitkrafttraining wirkte sich außerdem positiv auf Aufmerksamkeits- und Arbeitsgedächtnisleistungen bei den leicht betroffenen und noch nicht dementen Parkinson-Patienten positiv aus (5). Die Kraft der Rumpfextensoren ist bereits im Frühstadium der Erkrankung reduziert und geht mit eingeschränkter Fähigkeit zur Rumpfaufrichtung einher. Durch ein Krafttraining kann die funktionell beeinträchtigende Sitz-zu-Stand-Instabilität gebessert werden (37). Das Aufstehen von einem Stuhl ist für viele Parkinson-Patienten sogar die anspruchsvollste, mechanische funktionelle Aufgabe, die es im Alltag zu bewältigen gilt (38). Dieser Bewegungsablauf sollte innerhalb der physiotherapeutischen Behandlung repetitiv und selektiv trainiert werden, denn die Muskelkraft der 


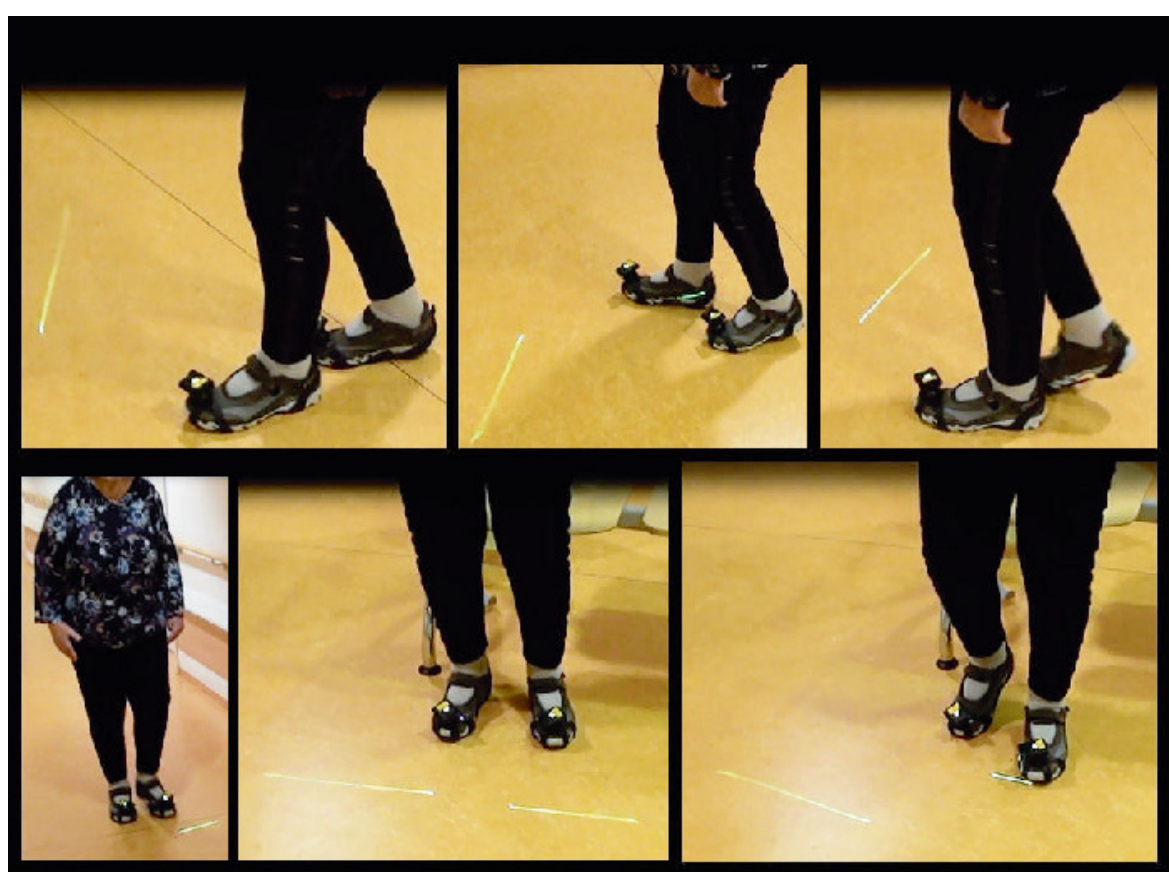

Abb. 2 LASER-Schuh zur Therapie und Training bei Freezing. Leicht auf die normalerweise getragenen Schuhe mit Gummilaschen anzubringende LASER-Quellen projizieren einen Strich auf den Boden nur beim Auftreten mit dem Vorfuß. Der sensorische Hinweisreiz kommt intermittierend zum Einsatz. Das Problem der Habituation wird damit umgangen (Aufnahmen von Video)
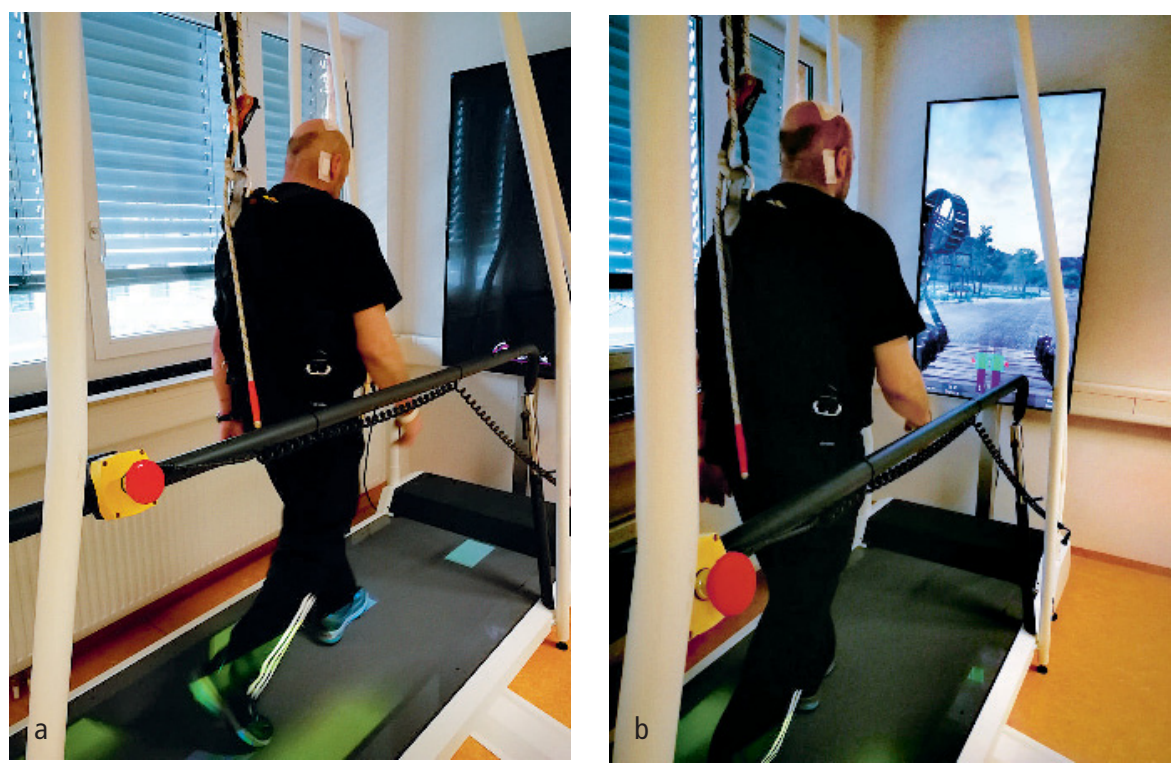

Abb. 3 Laufbandtraining kombiniert mit virtueller Realität: Trittziele können auf das Laufband projiziert werden A oder zu umgehende Hindernisse können über einen Bildschirm B dargestellt werden.

Hüftextensoren ist nachweislich reduziert und korreliert mit der Fähigkeit von einem Stuhl aufzustehen (39).

\section{Großamplitudiges}

Bewegungstraining

Das großamplitudige Bewegungstraining, in seiner speziellen Form das LSVT-BIG beinhaltet ein stereotypes und repetitives
Training mit möglichst großamplitudigen Bewegungen, das sich in erster Linie an körperlich fitte, leistungsbereite, motivierte und noch wenig betroffene Patienten richtet. Das Prinzip der Fokussierung auf die Lautstärke bei der LSVT-LOUD Stimmtherapie wird auf die Bewegungstherapie übertragen: Laut entspricht Groß (40).

\section{Therapie von Haltungsstörungen}

Kamptokormie, Pisa-Syndrom, Anterocollis sind bezüglich einer effektiven Physiotherapie bislang kaum untersucht. Ein Ausdauer- und Krafttraining der Rückenextensoren in Verbindung mit Erhalt ventraler Muskellängen durch muskuläre Entspannung und Dehnung, zeigte sich kürzlich auch bei extremen Haltungsstörungen als haltungsverbessernd (41). Als klinisch wirksam zeigt sich ein intensives Training der Hüftextensoren in Verbindung mit Perzeptionstraining der eigenen Haltung, unter Einsatz taktiler und visueller FeedbackMechanismen. Um aufrechtes Gehen im Alltag zu ermöglichen, sind Teleskopstöcke im Gebrauch. Durch diese können milde Formen der Kamptokormie kompensiert werden (42). Bei schwerer Kamptokormie ist ein hoher Rollator mit Unterarmschalen („Arthritisrollator“) effektiv, um zu trainieren, die Gehstrecke in aufrechter Haltung zu steigern und Rückenschmerzen zu reduzieren (43). Dass wie in einem Fallbericht beschrieben (44), eine Kamptokormie vom Tragen eines kleinen Rucksackes, durch den der Körperschwerpunkt nach hinten verlagert und das Aufrichten des Oberkörpers erleichtert werden soll, gelindert werden kann, ist von den Autoren bisher nicht beobachtet worden.

\section{Ergotherapie}

Traditionell orientiert sich die Ergotherapie in Abgrenzung zur Physiotherapie eher auf die Behandlung bzw. Kompensation von negativen Auswirkungen der Krankheit auf die Planung, Organisation und Durchführung von Alltagshandlungen. Wichtige Bereiche sind:

- Kommunikation z. B. Schreiben per Hand und am Computer, Telefonieren, 
- Selbstversorgung, z. B. Ankleiden, Nahrungsaufnahme mit Messer und Gabel,

- Häusliches Leben, z. B. Mahlzeiten vorbereiten.

Bis in die 2010er-Jahre gab es für die Ergotherapie im Vergleich zu der Physiotherapie wenig Studien. Dann wurde in einer multizentrischen Studie mit 191 Parkinson-Patienten (Hoehn \& Yahr 1-3) die Wirksamkeit der multimodalen Ergotherapie bei IPS nach der niederländischen Leitlinie untersucht (45). Dabei erzielte die Interventionsgruppe (IG) signifikant größere Verbesserungen in der Ausführung subjektiv wichtiger Alltagstätigkeiten als die Kontrollgruppe (KG) (Standardbehandlung). Diese hochrangig veröffentlichte Studie konnte bei der Erstellung der S3-Leitlinien nicht mehr berücksichtigt werden, sodass Ergotherapie „nur“ den mit dem zweit höchsten Empfehlungsgrad B aufgeführt wird.

\section{Sprech-, Stimm und \\ Schlucktherapie}

Eine relevante Dysarthrophonie tritt bei über $80 \%$ der Parkinson-Patienten in späteren Stadien auf (46) Aber viele Patienten leiden häufig auch schon früh im Verlauf trotz guter Verständlichkeit an kommunikativen Einschränkungen, die unterschätzt werden: Durch die reduzierte Prosodie und die Hypophonation kommt es zur falschen Interpretation von Emotionen durch den Gesprächspartner. Speziell für die Stimmstörung von Parkinson-Patienten wurde ein Lautstärke-Training entwickelt, das Lee Silverman Voice Treatment (LSVT-Loud ${ }^{\mathrm{Ts}}$ ), benannt nach einer Patientin namens Lee Silverman. Trainiert wird die Stimmstärke, Stimmstabilität und Tonhöhenumfang bei gleichzeitiger Verlängerung der Tonhaltedauer. Durch einen deutlich erhöhten Krafteinsatz bei allen Übungen soll der Patient die Atem-, Stimm- und Artikulationsmuskulatur trainieren und seine Sprechlautstärke steigern.

Durch ständige Rückmeldung des Therapeuten, auch mit Hilfe von Lautstärkepegelmessern und Videoaufnahmen, lernt der Patient, die Wahrnehmung seiner eigenen Stimme zu „rekalibrieren“. Die Diskrepanz zwischen motorischer Intention und

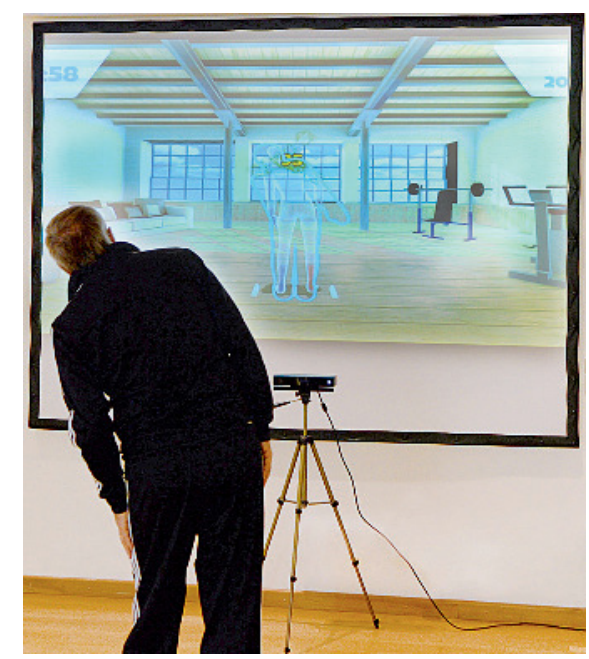

Abb. 4 Die Abbildung zeigt einen Patienten im Selbsttraining mit virtuellem Feedback in Form eines Avatars, der mit Hilfe der RGB-Kamera (KI$\mathrm{NECT}{ }^{\odot}$ ) dem Patienten dessen Körperhaltung spiegelt.

perzeptiv erreichtem Ziel soll damit überwunden werden. Die Therapie ist intensiv: Lege artis sollten über 4 Wochen 16 Einzelstunden durchgeführt werden. LSVT wurde LSVT mit reiner Atemtherapie verglichen. Dabei zeigten sich anhaltende Effekte nur in der LSVT-Gruppe mit den Ergebnissen einer deutlich größerer Lautstärke, Stimmumfang und Verständlichkeit: Verlaufsstudien über 6, 12 und 24 Monate nach Behandlung zeigen eine langfristig anhaltende Wirksamkeit von LSVT (47). Zwar wird LSVT-Loud ${ }^{\mathrm{m}}$ als Goldstandard in der Stimmtherapie betrachtet, aber große Studien, die die Evidenz eindeutig belegen fehlen bisher $(48,49)$. Deswegen erreicht diese Therapieform in den S3-Leitlinien „nur“ den zweit höchsten Empfehlungsgrad B.

\section{Schlucktherapie}

Auffällig ist bei Parkinson-Patienten in späteren Stadien die Diskrepanz zwischen dem gravierenden endoskopischen Befund und geringer subjektiver Beeinträchtigung durch die Schluckstörung. Wegen des schlechten Ansprechens der Dysphagie auf dopaminerge Medikation und der schlechten Prognose von Aspirationspneumonien bei Parkinson-Patienten kommt der Schlucktherapie eine wichtige Bedeutung $\mathrm{zu}$.

\section{Fazit für die Praxis}

Frisch diagnostizierten und leicht betroffenen Parkinson-Patienten sollte man gleich raten, angelehnt an die Empfehlungen der WHO, wöchentlich mindestens 150 Minuten moderate, körperliche Aktivität in zehnminütigen Einheiten anzustreben (21).

Spezifische aktivierende Therapien wie Kraft-, Gleichgewichts- und Ausdauertraining, auch im Rahmen komplexer motorischer Aktivitäten (z. B. Tai Chi, Tanz) und Ausdauersportarten (z. B. Nordic-Walking, Joggen, Fahrradfahren), ein lautstärkeorientiertes Training in der Logopädie und sein Pendant in der Physiotherapie das Training großer Bewegungsamplituden sind dann indiziert.

In späteren Stadien der ParkinsonKrankheit sind abhängig vom vorrangigen Störungsbild fokussierte auf Interventionen wie Gleichgewichts-, Antifreezing training (MAFT), Sturzprävention, Verbesserung der Autonomie (wie Essen, Trinken, Waschen und Ankleiden), Erhalt von Kommunikationsfähigkeit, evtl. auch bis hin zu computergestützten Kommunikatoren und die Verbesserung von Schlucken, insbesondere bei erhöhtem Aspirationsrisiko auszuwählen.

Systematische Studien zur Schlucktherapie bei Parkinson-Patienten finden sich wenige (50). Deswegen erreicht die Schlucktherapie in den S3-Leitlinien "nur" den zweit höchsten Empfehlungsgrad B. LSVT hat nach einer Untersuchung positive Effekte auf das Schlucken (51). Zwei kleinere randomisierte Studie zum Schucktraining bei Parkinson wurden durchgeführt, einmal mit videoassistiertem und zum anderen mit Elektrostimulation, die eine bessere Wirkung als konventionelle Schlucktherapie aufwiesen $(52,53)$. Am aussagekräftigsten ist eine Schein-interventionskontrollierte Studie an 60 ParkinsonPatienten zum Training der exspiratorischen Muskelkraft mit leichten bis mittelschweren Schluckstörungen, worunter eine signifikante Reduktion der Aspiration erzielt werden konnte (54). 


\section{Interessenkonflikt}

Vortragshonorare von AbbVie, Allergan, Archimedes, Bayer, Boston Scientific, Desitin, Grünenthal, Ipsen, Kyowa, Licher, Merz, Pfizer, Zambon.

\section{Literatur}

1. Ceballos-Baumann A, Ebersbach G. (Hrsg.) Aktivierende Therapien bei Parkinson-Syndromen. 3. Aufl. Stuttgart: Thieme 2017.

2. Tucha S. Psychotherapeutische Interventionen bei Morbus Parkinson aus klinischer Sicht. Nervenheilkunde 2018; 37: 257-263.

3. Clarke CE et al. Physiotherapy and Occupational Therapy vs No Therapy in Mild to Moderate Parkinson Disease: A Randomized Clinical Trial. JAMA Neurol 2016; 73(3): 291-299.

4. Tomlinson CL et al. Physiotherapy versus placebo or no intervention in Parkinson's disease. Cochrane Database Syst Rev 2013 (9): CD002817.

5. David FJ et al. Exercise improves cognition in Parkinson's disease: The PRET-PD randomized, clinical trial. Mov Disord 2015; 30(12): 1657-1663.

6. Cusso ME, Donald KJ, Khoo TK. The Impact of Physical Activity on Non-Motor Symptoms in Parkinson's Disease: A Systematic Review. Front Med (Lausanne) 2016; 3: 35.

7. Reynolds GO, Otto MW, Ellis TD, Cronin-Golomb A. The Therapeutic Potential of Exercise to Improve Mood, Cognition, and Sleep in Parkinson's Disease. Mov Disord 2016; 31(1): 23-38.

8. Petzinger GM, Fisher BE, McEwen S, Beeler JA, Walsh JP, Jakowec MW. Exercise-enhanced neuroplasticity targeting motor and cognitive circuitry in Parkinson's disease. Lancet Neurol 2013; 12(7): 716-726.

9. Kintz N, Petzinger GM, Jakowec MW. Treadmill exercise modifies dopamine receptor expression in the prefrontal cortex of the 1-methyl-4-phenyl-1,2,3,6-tetrahydropyridine-lesioned mouse model of Parkinson's disease. Neuroreport 2017; 28(15): 987-995.

10. Sasco AJ, Paffenbarger RS, Jr., Gendre I, Wing AL. The role of physical exercise in the occurrence of Parkinson's disease. Arch Neurol 1992; 49(4): 360-365.

11. Yang F et al. Physical activity and risk of Parkinson's disease in the Swedish National March Cohort. Brain 2015; 138(Pt 2): 269-275.

12. 12. LaHue SC, Comella CL, Tanner CM. The best medicine? The influence of physical activity and inactivity on Parkinson's disease. Mov Disord 2016; 31(10): 1444-1454.

13. 13. Hely MA, Morris JG, Reid WG, Trafficante R. Sydney Multicenter Study of Parkinsons disease: non-L-dopa-responsive problems dominate at 15 years. Mov Disord 2005; 20(2): 190-199.

14. DGN Ld. Idiopathisches Parkinson-Syndrom, Entwicklungsstufe: S3, Stand: 1. Januar 2016, Gültig bis: 31. Dezember 2020, AWMF-Registernummer: 030/010. https://wwwdgnorg/leitlinien/3219-030-010-idiopathisches-parkinsonsyndrom. 2016
15. Li F et al. Tai chi and postural stability in patients with Parkinson's disease. N Engl J Med 2012; 366(6): 511-519.

16. Raglio A. Music Therapy Interventions in Parkinson's Disease: The State-of-the-Art. Front Neurol 2015; 6: 185.

17. Elefant C, Baker FA, Lotan M, Lagesen SK, Skeie GO. The effect of group music therapy on mood, speech, and singing in individuals with Parkinsons disease - a feasibility study. J Music Ther 2012; 49(3): 278-302.

18. Shih LC, Piel J, Warren A, Kraics L, Silver A, Vanderhorst V, et al. Singing in groups for Parkinson's disease (SING-PD): a pilot study of group singing therapy for PD-related voice/speech disorders. Parkinsonism Relat Disord 2012; 18(5): 548-552.

19. Hackney ME, Earhart GM. Health-related quality of life and alternative forms of exercise in Parkinson disease. Parkinsonism Relat Disord 2009; 15(9): 644-648.

20. Ziegler K. E-health in der Physiotherapie bei Parkinson-Patienten. Nervenheilkunde 2015; 34 129-137.

21. Keus L et al. Europäische Physiotherapie-Leitlinie zum idiopatischen Parkinson-Syndrom Entwickelt von zwanzig europäischen Physiotherapieverbänden. http://wwwparkinsonnetinfo/guidelines. 2015.

22. Sturkenboom et al. Guidelines for Occupational Therapy in Parkinson's Disease Rehabilitation. http://wwwparkinsonnetinfo/media/ 14820461/ot_guidelines_final-npf_3_pdf. 2011.

23. Kalf $\mathrm{H}$ et al. Guidelines for Speech-Language Therapy in Parkinson's Disease. http://wwwparkinsonnetinfo/media/14829977/dutch_slp_guidelines-finalpdf. 2011.

24. NICE. Parkinson's disease in adults: diagnosis and management. Full guideline NG71. Methods, evidence and recommendations 2017 July.

25. George S, Tuschkan S. Ergotherapie. In: CeballosBauman A, Ebersbach G, editors. Aktivierende Therapien bei Parkinson-Syndromen, 3 Auflage. Stuttgart: Thieme 2017, 95-105.

26. Frank MJ, Seeberger LC, O'Reilly R C. By carrot or by stick: cognitive reinforcement learning in parkinsonism. Science 2004;306(5703):1940-3.

27. Nieuwboer A et al. Cueing training in the home improves gait-related mobility in Parkinson's disease: the RESCUE trial. J Neurol Neurosurg Psychiatry 2007;78(2):134-40.

28. Schroeteler FE, Ceballos-Baumann AO. Münchner Anti-Freezing-Training (MAFT). Reduzierung von Gangblockaden bei Parkinson. Nervenheilkunde 2016; 35: 198-204.

29. Fietzek UM, Schroeteler FE, Ziegler K, Zwosta J, Ceballos-Baumann AO. Randomized cross-over trial to investigate the efficacy of a two-week physiotherapy programme with repetitive exercises of cueing to reduce the severity of freezing of gait in patients with Parkinsons disease. Clin Rehabil 2014; 28(9): 902-911.

30. Fietzek UM, Zwosta J, Schroeteler FE, Ziegler K, Ceballos-Baumann AO. Levodopa changes the severity of freezing in Parkinson's disease. Parkinsonism Relat Disord 2013.

31. Ziegler K, Schroeteler F, Ceballos-Baumann AO, Fietzek UM. A new rating instrument to assess fes- tination and freezing gait in Parkinsonian patients. Mov Disord 2010; 25(8): 1012-1018.

32. Ziegler K. Stürze bei Patienten mit leichtem und fortgeschrittenem Parkinsonsyndrom und ihre Prophylaxe durch Physiotherapie. Nervenheilkunde 2018; 37: 249-256.

33. Mirelman A et al. Addition of a non-immersive virtual reality component to treadmill training to reduce fall risk in older adults (V-TIME): a randomised controlled trial. Lancet 2016; 388(10050): 1170-1182.

34. Jobges M, Heuschkel G, Pretzel C, Illhardt C, Renner C, Hummelsheim H. Repetitive training of compensatory steps: a therapeutic approach for postural instability in Parkinson's disease. J Neurol Neurosurg Psychiatry 2004; 75(12): 1682-1687.

35. Barry G, Galna B, Rochester L. The role of exergaming in Parkinson's disease rehabilitation: a systematic review of the evidence. J Neuroeng Rehabil 2014; 11: 33 .

36. Corcos DM et al. A two-year randomized controlled trial of progressive resistance exercise for Parkinson's disease. Mov Disord 2013; 28(9): 1230-1240.

37. Dibble LE, Hale TF, Marcus RL, Droge J, Gerber JP, LaStayo PC. High-intensity resistance training amplifies muscle hypertrophy and functional gains in persons with Parkinson's disease. Mov Disord 2006; 21(9): 1444-1452.

38. Nikfekr E, Kerr K, Attfield S, Playford DE. Trunk movement in Parkinson's disease during rising from seated position. Mov Disord 2002; 17(2): 274-282.

39. Inkster LM, Eng JJ. Postural control during a sitto-stand task in individuals with mild Parkinson's disease. Exp Brain Res 2004; 154(1): 33-38.

40. Schroeteler F. LSVT-BIG: Motorisches Trainingskonzept für Menschen mit Parkinson. Nervenheilkunde 2018; 37: 243-248.

41. Bartolo M et al. Four-week trunk-specific rehabilitation treatment improves lateral trunk flexion in Parkinson's disease. Mov Disord 2010; 25(3): 325-331.

42. Schroeteler F, Ziegler K. Physiotherapie bei Parkinson-Syndromen. In: Ceballos-Bauman A, Ebersbach G, editors. Aktivierende Therapien bei Parkinson-Syndromen. Stuttgart: Thieme 2017, 95-105.

43. Schroeteler FE, Fietzek UM, Ziegler K, CeballosBaumann AO. Upright posture in parkinsonian camptocormia using a high-frame walker with forearm support. Mov Disord 2011; 26(8): $1560-1561$

44. Gerton BK, Theeler B, Samii A. Backpack treatment for camptocormia. Mov Disord 2010; 25(2): 247-248.

45. Sturkenboom IH et al. Efficacy of occupational therapy for patients with Parkinson's disease: a randomised controlled trial. Lancet Neurol 2014; 13(6): 557-566.

46. Hely MA, Reid WG, Adena MA, Halliday GM, Morris JG. The Sydney multicenter study of Parkinson's disease: the inevitability of dementia at 20 years. Mov Disord 2008; 23(6): 837-844.

47. Ramig LO et al. Intensive voice treatment (LSVT) for patients with Parkinson's disease: a 2 year follow up. J Neurol Neurosurg Psychiatry 2001; 71(4): 493-498. 
48. Piechler K, Maillen G. Die Sprech- und Stimmstörung beim idiopathischen Parkinson-Syndrom. In Ceballos-Bauman A, Ebersbach G, editors. Aktivierende Therapien bei Parkinson-Syndromen. Stuttgart: Thieme 2017, 41-5.

49. Herd CP et al. Comparison of speech and language therapy techniques for speech problems in Parkinson's disease. Cochrane Database Syst Rev 2012(8): CD002814.

50. Wagner-Sonntag E. Schluckstörungen bei Parkinson-Syndromen. In: Ceballos-Bauman A, Ebers- bach G (Hrsg.) Aktivierende Therapien bei Parkinson-Syndromen. Stuttgart: Thieme 2017, 49-57.

51. Sharkawi AE et al. Swallowing and voice effects of Lee Silverman Voice Treatment (LSVT): a pilot study. J Neurol Neurosurg Psychiatry 2002; 72(1): 31-36.

52. Baijens LW, Speyer R, Passos VL, Pilz W, van der Kruis J, Haarmans S, et al. Surface electrical stimulation in dysphagic Parkinson patients: a randomized clinical trial. Laryngoscope 2013; 123(11): E38-E44.
53. Manor Y, Mootanah R, Freud D, Giladi N, Cohen JT. Video-assisted swallowing therapy for patients with Parkinson's disease. Parkinsonism Relat Disord 2013; 19(2): 207-211.

54. Troche MS et al. Aspiration and swallowing in Parkinson disease and rehabilitation with EMST: a randomized trial. Neurology 2010; 75(21): 1912-1919. 